\title{
Kinetics of Pulmonary Gas Exchange During and While Recovering From Exercise in Patients After Anterior Myocardial Infarction
}

\author{
Noritaka Shimizu, MD; Akira Koike, MD*; Yoshiharu Koyama, MD; \\ Kazuo Kobayashi, MD; Fumiaki Marumo, MD; Michiaki Hiroe, MD
}

\begin{abstract}
The effect of exercise intensity on gas exchange kinetics was investigated during exercise and recovery, as well as the relationship between the kinetics during exercise and recovery. Twenty-three patients with a history of anterior myocardial infarction performed low-intensity $(38.7 \pm 8.3 \mathrm{~W})$ and high-intensity $(68.8 \pm 15.0 \mathrm{~W})$ exercise for 6 min. The time constants of oxygen uptake $\left(\dot{\mathrm{V}}_{2}\right)$, carbon dioxide output $\left(\dot{\mathrm{V} C \mathrm{O}_{2}}\right)$ and minute ventilation $(\dot{\mathrm{V} E})$ were significantly prolonged during high intensity exercise compared with low-intensity exercise $(61.2 \pm 8.6$ vs $52.3 \pm 10.3 \mathrm{~s}, \mathrm{p}<0.005$ for the time constant of $\dot{\mathrm{V}} \mathrm{O}_{2}$ ). The time constant of $\dot{\mathrm{V}}_{2}$ was similar during exercise and during recovery from exercise of high $(61.2 \pm 8.6$ vs $66.2 \pm 12.2 \mathrm{~s})$ as well as low intensity $(52.3 \pm 10.3$ vs $55.0 \pm 10.1 \mathrm{~s}$ ). However, the time constants of $\mathrm{VCO}_{2}$ and heart rate were significantly shorter during recovery than during exercise. The time constants of $\dot{\mathrm{V}} \mathrm{CO}_{2}$ and $\dot{\mathrm{V}} \mathrm{E}$ were significantly longer than that of $\dot{\mathrm{VO}}_{2}$ during both exercise and recovery. In the present study, it was found that (1) the gas exchange kinetics were influenced by the intensity of exercise; (2) the kinetics during recovery did not necessarily reflect the kinetics during exercise except for $\dot{\mathrm{V}}_{2}$; and (3) the kinetics of $\dot{\mathrm{V}} \mathrm{CO}_{2}$ and $\dot{\mathrm{V}} \mathrm{E}$ were delayed as compared with the $\dot{\mathrm{V}}_{2}$ kinetics. These characteristics should be taken into account when using gas exchange kinetics to estimate cardiopulmonary responses to exercise in patients with left ventricular dysfunction. (Jpn Circ J 1999; 63: 459-466)
\end{abstract}

Key Words: Carbon dioxide output; Oxygen uptake; Time constant; Ventilation

$\mathbf{T}$ he increase in pulmonary oxygen uptake $\left(\dot{\mathrm{V}}_{2}\right)$ at the onset of exercise reflects muscular $\dot{\mathrm{VO}}_{2}$ and can be used to evaluate the rapidity of cardiovascular adaptation to exercise ${ }^{1-3}$ The kinetics of $\dot{\mathrm{V}}_{2}$ are assessed by the time constant determined by fitting an exponential equation to the response of $\dot{\mathrm{V}}_{2}$ to exercise ${ }^{3-7}$ The time constant of $\dot{\mathrm{V}}_{2}$ is correlated with the peak $\dot{\mathrm{VO}}_{2}$ obtained from symptom-limited incremental exercise in cardiac patients and can be determined without maximal effort?.8 Thus, the time constant of $\dot{\mathrm{VO}}_{2}$ during exercise is used in the clinical setting to assess the severity of disease and to evaluate the efficacy of therapeutic interventions in patients with cardiovascular disease,, $7-13$ Because the kinetics of carbon dioxide output $\left(\dot{\mathrm{VCO}}_{2}\right)$ would be related to the degree of lactic acidosis, and the kinetics of minute ventilation (VE) must be influenced by the degree of exercise-induced hyperventilation, time constants of these variables must also be useful for estimating the impairment of the cardiopulmonary responses to exercise $3,6,9,11,12,14$

The optimal exercise intensity for assessing time constants has not been determined. We previously determined the time constant during low-intensity, constant work-rate exercise at approximately $50 \mathrm{~W}$ and during recovery, 37 Other studies have suggested that gas exchange kinetics obtained during recovery after maximal exercise

(Received November 17, 1998; revised manuscript received March 8, 1999; accepted March 18, 1999)

Second Department of Internal Medicine and $*$ Department of Critical Care Medicine, Tokyo Medical and Dental University, Tokyo, Japan Mailing address: Akira Koike, MD, Department of Critical Care Medicine, Tokyo Medical and Dental University, 5-45 Yushima 1chome, Bunkyo-ku, Tokyo 113-8519, Japan are useful for assessing impaired circulatory response!1,12 However, quantitative data on the influence of exercise intensity on gas exchange kinetics in patients with left ventricular dysfunction are limited. If the exercise intensity affects kinetics, it could be misleading to compare time constants measured during exercise at different intensities or those obtained during recovery after incremental exercise terminated at different work rates.

$\dot{\mathrm{V}} \mathrm{O}_{2}$ kinetics during recovery from low-intensity constant work-rate exercise are assumed to reflect $\dot{\mathrm{V}}_{2}$ kinetics during exercise, ${ }^{1,7}$ However, the relationship between other gas exchange kinetic parameters during exercise and recovery has not been confirmed. Furthermore, the relationship among $\dot{\mathrm{V}}_{2}, \dot{\mathrm{V}}_{2}$, and $\dot{\mathrm{V}} \mathrm{E}$ kinetics, which is important for the evaluation of the coupling of pulmonary gas exchange and metabolism, has not been established.

In the present study, patients with anterior myocardial infarction performed both low- and high-intensity constant work-rate exercise. Breath-by-breath respiratory gases and heart rate (HR) were measured noninvasively. We investigated the effect of exercise intensity on the kinetics of $\dot{\mathrm{V}}_{2}$, $\dot{\mathrm{V}} \mathrm{CO}_{2}, \dot{\mathrm{V}} \mathrm{E}$, and HR during exercise and recovery, the relationship between exercise and recovery kinetics, and the relationship between the kinetics of pulmonary gas exchange and HR.

\section{Methods}

Study Patients

We studied 23 patients with a history of anterior myocardial infarction (New York Heart Association func- 

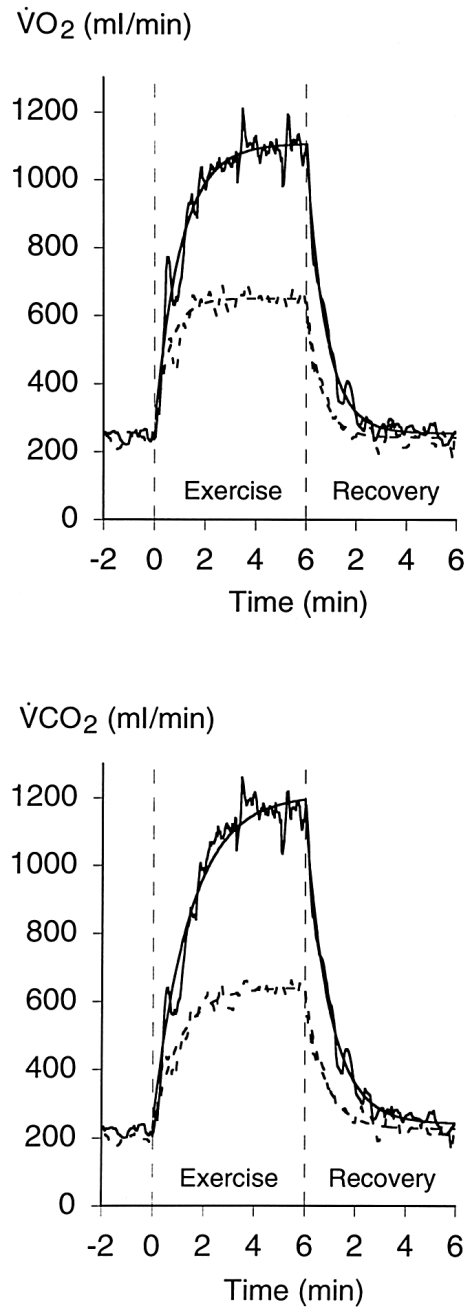

HR (bpm)
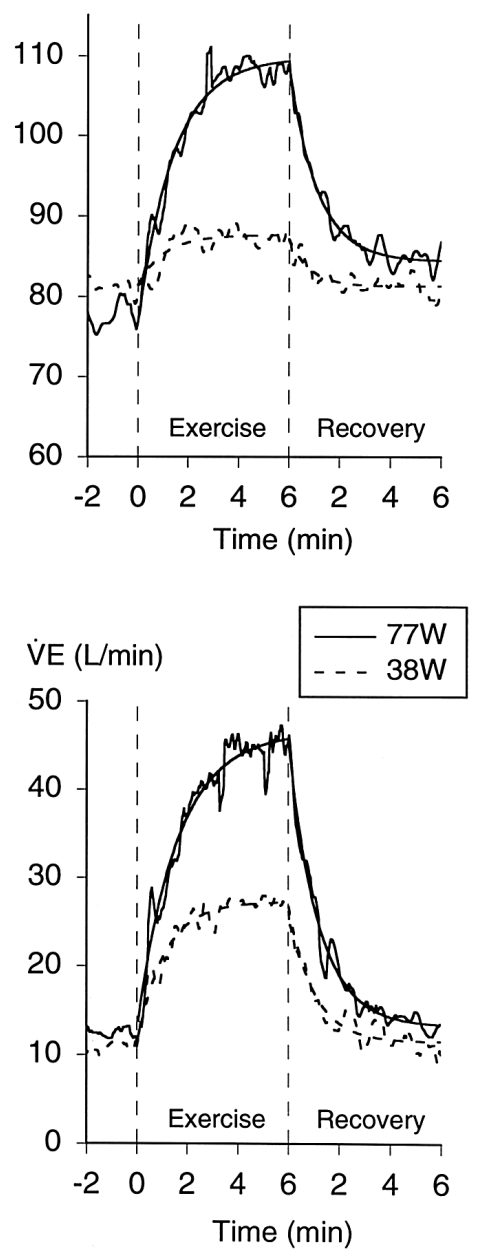

Fig 1. Changes in pulmonary gas exchange and heart rate during exercise and recovery $(38 \mathrm{~W}$ and $77 \mathrm{~W}$ exercise) in a 64-year-old male patient. The computer-derived lines of the best fit to a single exponential model of the response are also shown. HR, heart rate; $\dot{\mathrm{V}} \mathrm{CO}_{2}$, carbon dioxide output; $\dot{\mathrm{V}} \mathrm{E}$, minute ventilation; $\dot{\mathrm{VO}}_{2}$, oxygen uptake; bpm, beats per minute. tional classes I-II). Twenty patients were men and 3 were

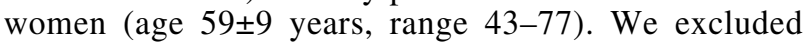
patients who experienced chest pain and/or who needed sublingual nitrates during maximal exercise and those with documented lung disease. No patient had had a myocardial infarction in the month preceding study enrollment. Seven patients out of the 23 had left ventricular aneurysm diagnosed by echocardiography and/or contrast ventriculography. All subjects were sedentary and had not participated in a regular exercise program in the past year. At the time of the study, they were clinically stable and in sinus rhythm. No patient revealed ventricular tachycardia on Holter electrocardiogram. Although digitalis, nitrates, and calcium antagonists were prescribed in 3, 19 and 12 patients, respectively, all medications were withheld for at least $24 \mathrm{~h}$ before the study. Beta-adrenergic blocking agents were withheld for 7 days to avoid their influence on HR kinetics 15,16 The nature and purpose of the study and risks involved were explained to the patients and informed consent was obtained from each prior to enrollment.

\section{Exercise Protocol}

Patients performed relatively low-intensity and highintensity exercise at a constant work rate in the upright position using an electromagnetically braked cycle ergometer (Siemens-Elema 930, Siemens Elema AB, Sweden or WLP-400, Lode, Holland). The interval between the tests was approximately $30 \mathrm{~min}$. After resting for $2 \mathrm{~min}$, subjects performed $6 \mathrm{~min}$ of exercise followed by a 6-min recovery period, during which subjects remained in the upright position on the ergometer without cooling down.

To obviate the energetic effect of inertia while the flywheel was accelerated at the initiation of exercise, the flywheel was driven manually at approximately $100 \mathrm{rpm}$ before the start of exercise. During this period, the patient's feet were placed on the box between the pedals at the same height as the pedals. The feet were then moved by a technician onto the pedals, and the patient began pedaling within a few seconds when the speed of the flywheel reached 60 rpm.

The work rates selected as low and high intensities were determined based on each subject's gas exchange (anaerobic) threshold ${ }^{17-19}$ and the maximal work rate obtained during a preliminary incremental exercise test. The lowintensity work rate corresponded to $80 \%$ of the work rate at the gas exchange threshold. To determine the work rate for high-intensity exercise, we calculated the difference between the work rate at peak exercise and the gas exchange threshold and then increased the work rate at the gas exchange threshold by $40 \%$ of that difference. The preliminary incremental exercise test was performed within 2 weeks of the day of study.

Breath-by-breath $\dot{\mathrm{V}} \mathrm{O}_{2}, \dot{\mathrm{V}} \mathrm{CO}_{2}$, and $\dot{\mathrm{V}} \mathrm{E}$ were measured throughout the test until $6 \mathrm{~min}$ of recovery using a Respiromonitor RM 300 (Minato Medical Science, Osaka, 
Table 1 Hemodynamic and Respiratory Gas Data at Rest and During 6 min of Exercise

\begin{tabular}{lccc}
\hline \hline & \multirow{2}{*}{ Rest } & \multicolumn{2}{c}{ Exercise } \\
\cline { 3 - 4 } & & Low intensity & High intensity \\
\hline Work rate $(\mathrm{W})$ & & $38.7 \pm 8.3$ & $68.8 \pm 15.0^{*}$ \\
Heart rate $($ beats/min) & $77.9 \pm 12.3$ & $108.3 \pm 19.4$ & $138.0 \pm 22.2^{*}$ \\
Systolic blood pressure $(\mathrm{mmHg})$ & $125.3 \pm 15.0$ & $157.7 \pm 23.7$ & $176.8 \pm 23.2^{*}$ \\
Diastolic blood pressure $(\mathrm{mmHg})$ & $79.7 \pm 11.5$ & $86.7 \pm 15.6$ & $97.5 \pm 17.2^{*}$ \\
$\dot{\mathrm{V}} \mathrm{O}_{2}(\mathrm{ml} / \mathrm{min})$ & $222.8 \pm 34.0$ & $730.9 \pm 120.0$ & $1063.0 \pm 210.7^{*}$ \\
$\dot{\mathrm{VCO}}(\mathrm{ml} / \mathrm{min})$ & $180.4 \pm 30.8$ & $694.6 \pm 122.6$ & $1107.0 \pm 229.3^{*}$ \\
$\dot{V} E(\mathrm{~L} / \mathrm{min})$ & $10.3 \pm 2.0$ & $28.7 \pm 6.0$ & $45.0 \pm 9.8^{*}$ \\
Lactate $(\mathrm{mmol} / \mathrm{L})$ & $1.3 \pm 0.6$ & $2.8 \pm 1.3$ & $5.6 \pm 2.8^{*}$ \\
\hline
\end{tabular}

Values are the mean $\pm S D$. The venous lactate concentration at low- and high-intensity exercise was obtained 2 min after exercise. ${ }^{*} p<0.05$ vs low-intensity test by a paired student's $t$-test.

Table 2 Time Constants (TC) of Heart Rate and Respiratory Gases During Exercise and Recovery

\begin{tabular}{lccccc}
\hline \hline \multirow{2}{*}{ Time constant $(s)$} & \multicolumn{2}{c}{ During exercise } & & \multicolumn{2}{c}{ During recovery } \\
\cline { 2 - 3 } \cline { 5 - 5 } & Low intensity & High intensity & & Low intensity & High intensity \\
\hline$\dot{V} \mathrm{O}_{2}$ & $52.3 \pm 10.3$ & $61.2 \pm 8.6^{*}$ & & $55.0 \pm 10.1$ & $66.2 \pm 12.2^{*}$ \\
$\dot{V} C O_{2}$ & $78.5 \pm 18.2$ & $95.9 \pm 19.5^{*}$ & & $66.9 \pm 14.2^{\dagger}$ & $82.7 \pm 18.0^{* \dagger}$ \\
$\dot{V} E$ & $74.6 \pm 16.1$ & $111.5 \pm 31.3^{*}$ & & $72.4 \pm 20.5$ & $81.1 \pm 21.2^{\dagger}$ \\
Heart rate & $59.2 \pm 38.3$ & $99.3 \pm 41.8^{*}$ & & $34.9 \pm 13.2^{\dagger}$ & $68.7 \pm 21.0^{* \dagger}$ \\
\hline
\end{tabular}

Values are the mean $\pm S D$.

${ }^{*} p<0.005 v s$ TC of the low-intensity test; ${ }^{\dagger} p<0.05$ vs TC during exercise by a paired student's t-test.

Japan), as previously described 3,7 The system was carefully calibrated before each study.

A 12-lead electrocardiogram was monitored continuously (System ML-8000, Fukuda Denshi Co, Ltd, Tokyo, Japan) and recorded breath-by-breath, simultaneously with measurements of respiratory gases in 21 patients. For the remaining 2 patients, HR was recorded every minute, not on a breath-by-breath basis, for both low- and high-intensity exercise. We excluded the time constants of HR of these subjects from the analysis.

Cuff blood pressures were obtained every minute with an automatic manometer (STBP-680F, Collin Denshi, Aichi, Japan) ${ }^{20}$ Superficial forearm vein blood was obtained before and $2 \mathrm{~min}$ after the exercise test for measurement of lactate (enzymatic method) ${ }^{21}$ to determine if gas exchange kinetics were associated with the degree of lactic acidosis.

\section{Data Analysis}

The 6-min values for HR and respiratory gases were determined as the average values obtained between 330 and $360 \mathrm{~s}$ during the exercise period and the recovery period. A 5-point moving average of the breath-by-breath data was used to evaluate the kinetics of each variable?,7 The time constants of these kinetics during exercise were determined by fitting a single exponential function to each response starting at the onset of exercise, with the resting value defined as the baseline (Fig 1),-7 using the following equation:

$$
\mathrm{Y}(\mathrm{t})=\mathrm{Y}(\mathrm{b})+\mathrm{A}(1-\mathrm{e}-\mathrm{t} / \mathrm{t})
$$

where $\mathrm{Y}(\mathrm{t})$ is the $\mathrm{HR}, \dot{\mathrm{V}} \mathrm{O}$ 2, $\dot{\mathrm{V}} \mathrm{CO}$, , or $\dot{\mathrm{V}} \mathrm{E}$ at time $\mathrm{t}$; $\mathrm{Y}(\mathrm{b})$ is the baseline value of that variable at rest; $\mathrm{A}$ is the amplitude of the response (increment above baseline), and $\mathrm{T}$ is the time constant. The time constant (T ) and amplitude (A) were derived by nonlinear regression analysis using leastsquares and iterative techniques ${ }^{3,5-7}$ with a BMDP statistical software package?2 ${ }^{22}$ The time constants during recovery from exercise were determined in the same manner, using the values at the end of exercise as the baseline?

\section{Statistics}

Data are presented as the mean $\pm \mathrm{SD}$. Low- and highintensity exercise time constants of HR and gas exchange kinetics were compared by the Student's paired t-test. Linear regression analysis was used to determine correlations between time constants and the venous lactate concentration. Differences were considered statistically significant when the 2-tailed p-value was less than 0.05 .

\section{Results}

The mean peak $\dot{\mathrm{V}}_{2}$ and gas exchange threshold obtained during preliminary incremental exercise were $20.2 \pm 3.7$ and $13.1 \pm 1.7 \mathrm{ml} \mathrm{min}^{-1} \mathrm{~kg}^{-1}$, respectively. The mean work rate was $38.7 \pm 8.3 \mathrm{~W}$ for low-intensity exercise and $68.8 \pm 15.0 \mathrm{~W}$ for high-intensity exercise (Table 1). All subjects performed both tests without adverse effects.

\section{Effects of Exercise Intensity on Heart Rate and Gas Exchange Kinetics}

The time constants of $\dot{\mathrm{V}} 2$, $\dot{\mathrm{V}} \mathrm{CO}_{2}, \dot{\mathrm{V}} \mathrm{E}$, and HR were significantly prolonged during high-intensity exercise as compared with low-intensity exercise (Table 2). The time constants of $\dot{\mathrm{V}} 2, \dot{\mathrm{V}} \mathrm{CO}_{2}$, and HR were significantly prolonged during recovery from high-intensity exercise as compared with recovery from low-intensity exercise, although the difference between the time constant of $\dot{V} E$ during recovery from high-intensity exercise and that of recovery from low-intensity exercise did not reach a statistical significance $(\mathrm{p}=0.13)$.

\section{Relationship Between Gas Exchange Kinetics During Exercise and Recovery}

The time constant of $\dot{\mathrm{V}} \mathrm{O}_{2}$ during recovery from exercise was significantly correlated with the time constant of $\dot{\mathrm{V}} \mathrm{O}_{2}$ 

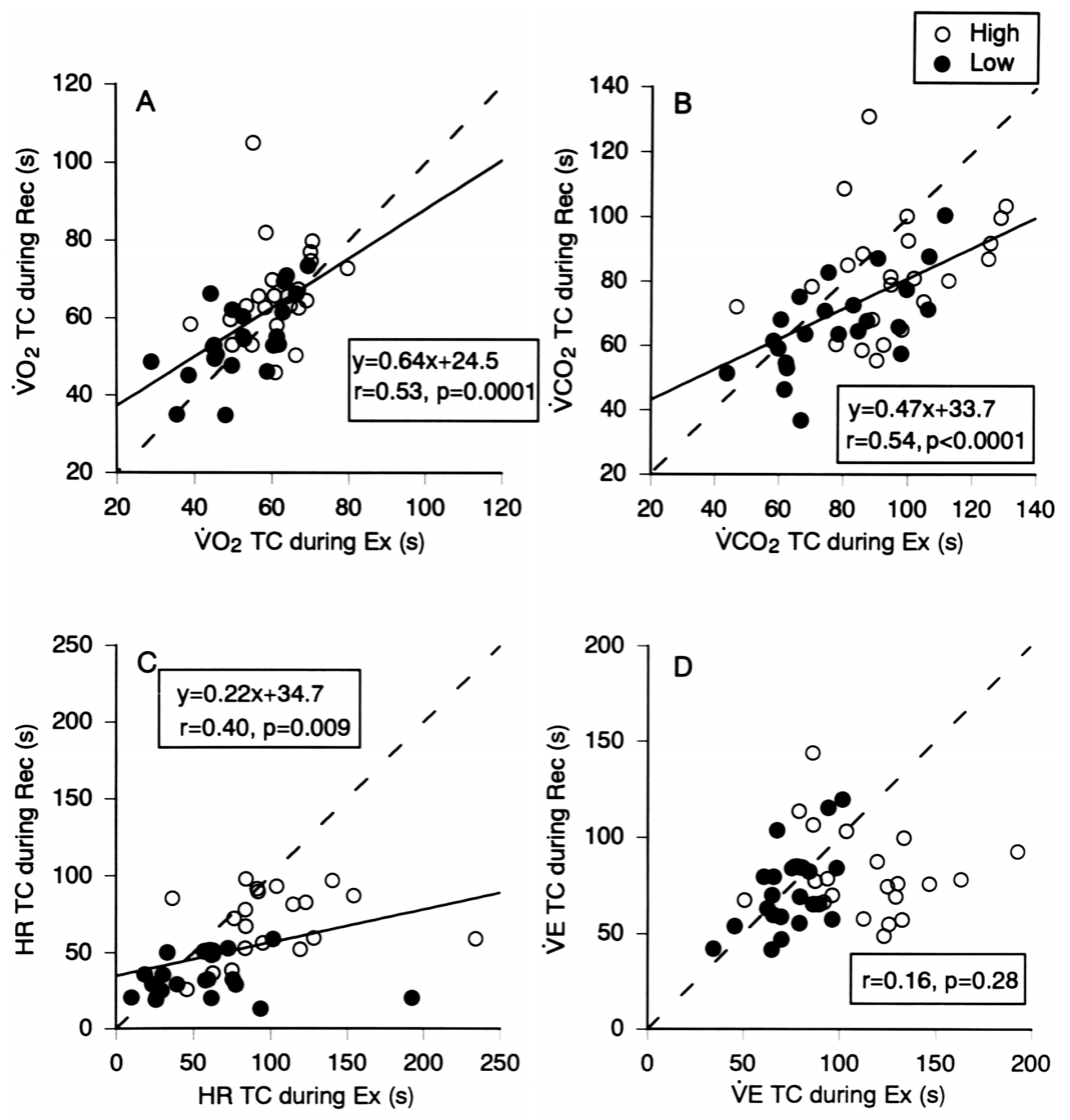

Fig 2. Relationship between gas exchange and heart rate kinetics during exercise (Ex) and during the recovery from exercise (Rec). Solid lines are the regression lines and broken lines are the lines of identity. HR, heart rate; TC, time constant; $\dot{\mathrm{V}} \mathrm{CO}_{2}$, carbon dioxide output; $\dot{\mathrm{V}} \mathrm{E}$, minute ventilation; $\dot{\mathrm{VO}} 2$, oxygen uptake.
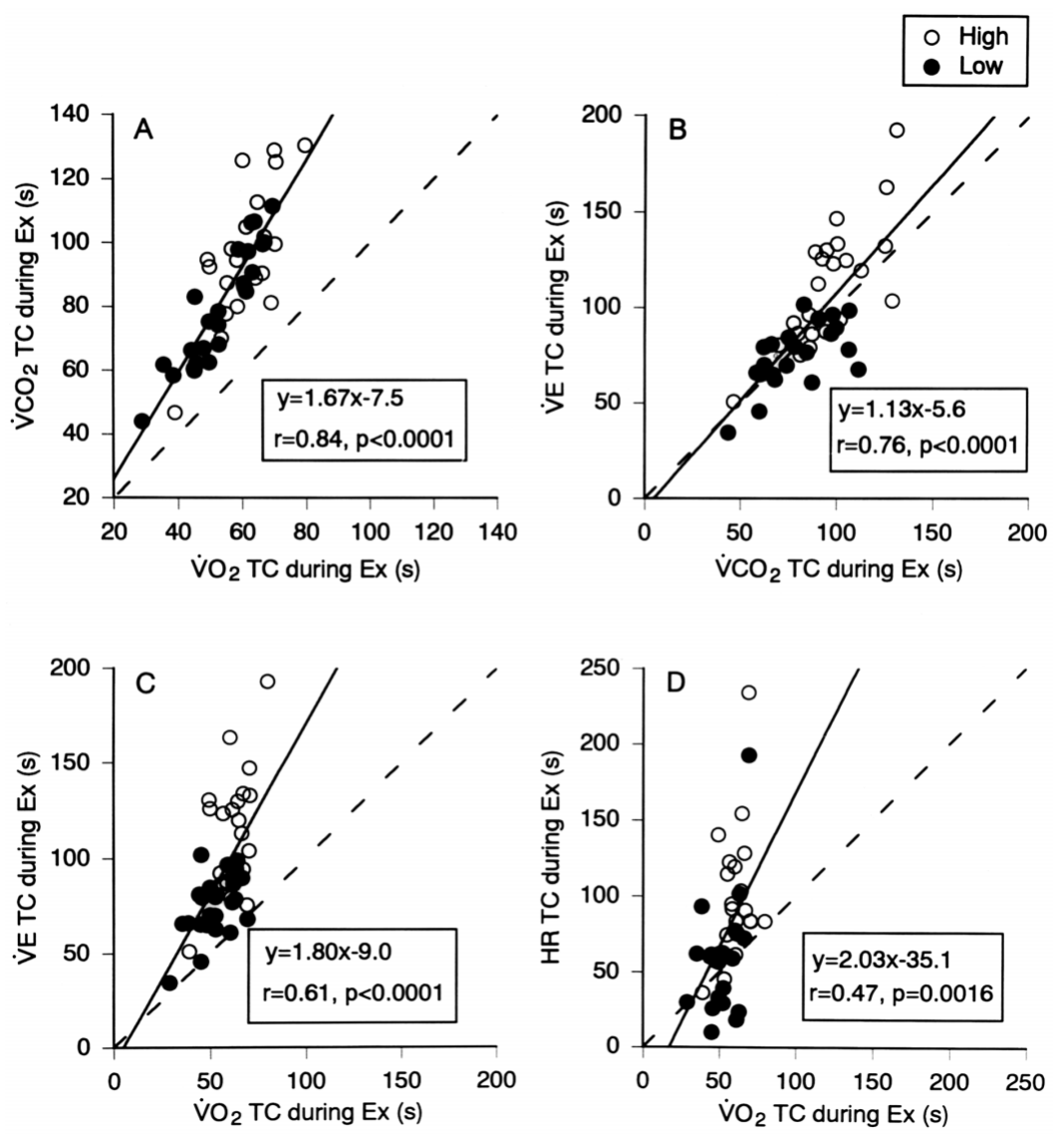

Fig 3. Relationship between gas exchange and heart rate kinetics during low- and high-intensity exercise. Solid lines are the regression lines for all of the data and broken lines are the lines of identity. HR, heart rate; TC, time constant; $\dot{\mathrm{V}} \mathrm{CO}_{2}$, carbon dioxide output; $\grave{\mathrm{V} E}$, minute ventilation; $\dot{\mathrm{V}}$ 2, oxygen uptake. 

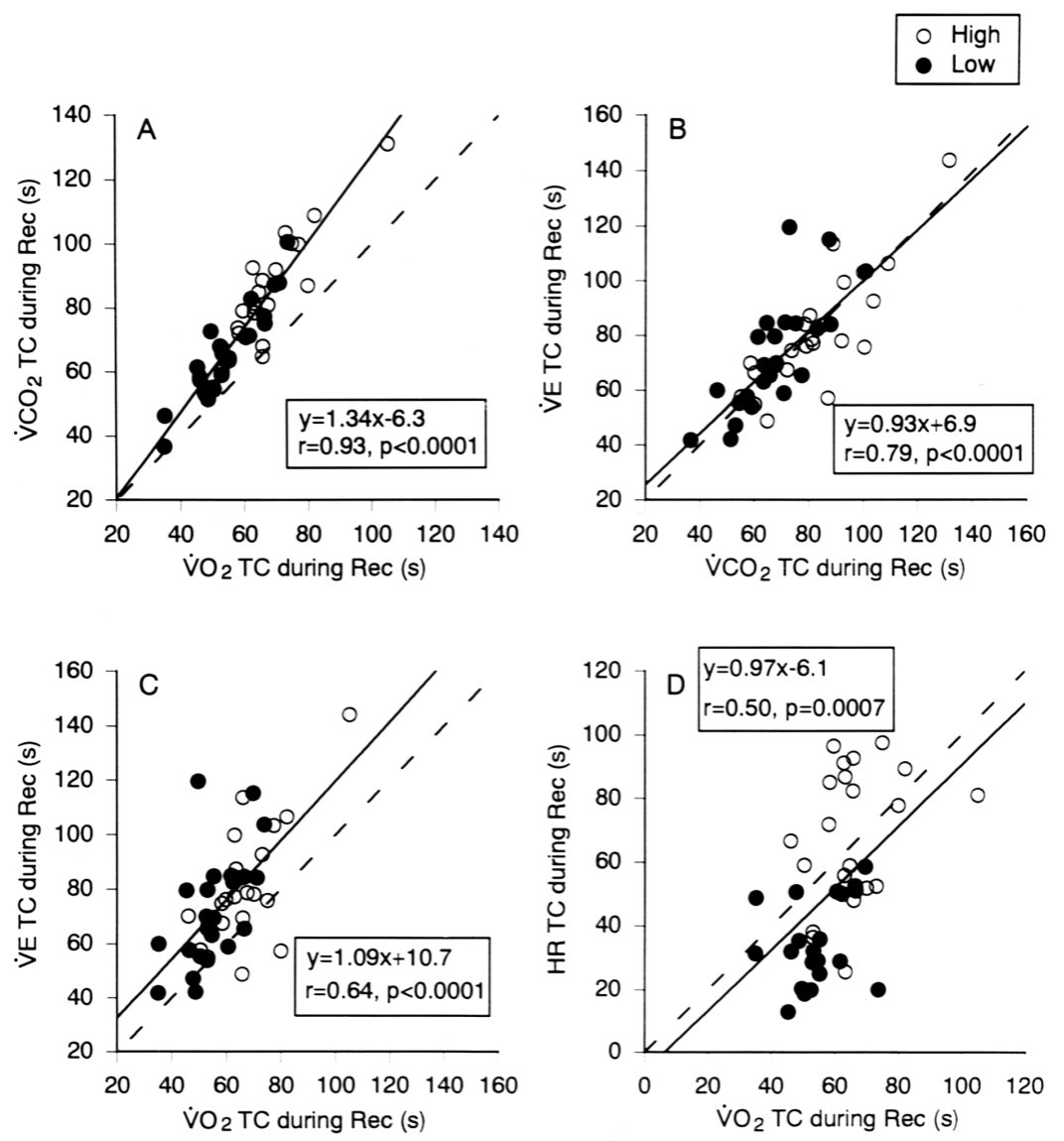

Fig 4. Relationship between gas exchange and heart rate kinetics during recovery from low- and high-intensity exercise. Solid lines are the regression lines for all of the data and broken lines are the lines of identity. HR, heart rate; TC, time constant; $\dot{\mathrm{V}} \mathrm{CO}_{2}$, carbon dioxide output; $\dot{\mathrm{V}} \mathrm{E}$, minute ventilation; $\dot{\mathrm{V}} \mathrm{O}_{2}$, oxygen uptake.

during exercise ( $\mathrm{r}=0.53$, Fig 2A). There was no significant difference between these parameters at exercise of low or high intensity (Table 2). There was also a significant correlation between the time constant of $\dot{\mathrm{V}} \mathrm{CO}_{2}$ during exercise and recovery ( $\mathrm{r}=0.54$, Fig 2B). However, the time constant of $\dot{\mathrm{V}} \mathrm{CO}_{2}$ was significantly shorter during recovery than during exercise for both the low- and high-intensity exercise $(p<0.05$, Table 2$)$. The time constant of HR was also shorter during recovery than during exercise for both lowand high-intensity exercise $(p<0.05)$; there was a significant, but weak correlation between these parameters $(\mathrm{r}=0.40$, Fig $2 \mathrm{C})$. The time constant of $\dot{\mathrm{V} E}$ during recovery was not correlated with the time constant of VE during exercise (Fig 2D).

\section{Relationship Between Gas Exchange and \\ Heart Rate Kinetics During Exercise}

$\dot{\mathrm{V}} \mathrm{CO}_{2}$ kinetics were significantly slower than $\dot{\mathrm{V}}_{2}$ kinetics at both low- and high-intensity exercise $(\mathrm{p}<0.01$, Fig $3 \mathrm{~A}$ ). The time constants of $\dot{\mathrm{V}} \mathrm{CO}_{2}$ and $\dot{\mathrm{V}} \mathrm{O}_{2}$ showed a strong correlation for exercise of both low- and high-intensity $(\mathrm{r}=0.84)$. There was a significant positive correlation between the kinetics of $\dot{\mathrm{V}} \mathrm{CO}_{2}$ and those of $\dot{\mathrm{VE}}(\mathrm{r}=0.76$, Fig 3B), although VE kinetics were slower than $\dot{\mathrm{V}} \mathrm{CO}_{2}$ kinetics for high-intensity exercise $(\mathrm{p}<0.01)$. Although the time constant of $\dot{V} E$ was significantly longer than that of $\dot{\mathrm{V}} \mathrm{O}_{2}$ for both tests $(\mathrm{p}<0.01)$, these parameters were significantly correlated ( $r=0.61$, Fig 3C). There was a weak correlation between the time constant of $\mathrm{HR}$ and that of $\dot{\mathrm{VO}}_{2}$ $(r=0.47$, Fig 3D). The venous lactate concentration obtained 2 min after exercise was positively correlated with the time constant of $\dot{V} E$ during exercise $(r=0.69, p<0.001)$, but was weakly correlated with the time constants of $\dot{\mathrm{V} O} 2$ $(\mathrm{r}=0.45, \mathrm{p}<0.01)$ and $\dot{\mathrm{V} C O} 2(\mathrm{r}=0.48, \mathrm{p}<0.01)$.

\section{Relationship Between Gas Exchange and Heart}

Rate Kinetics During Recovery From Exercise

The relationships among gas exchange kinetics during recovery from exercise resembled those observed during exercise. There were significant positive correlations among the time constants of $\dot{\mathrm{VO}}_{2}, \dot{\mathrm{V}} \mathrm{CO}_{2}$, and $\dot{\mathrm{V} E}$ during recovery from exercise ( $\mathrm{r}=0.64$ to 0.93 , Fig 4 ), although the time constants of $\dot{\mathrm{V}} \mathrm{CO}_{2}$ and $\dot{\mathrm{V}} \mathrm{E}$ were significantly longer than the time constants of $\dot{\mathrm{V}} \mathrm{O}_{2}$ for both low- and high-intensity exercise $(p<0.01)$. There was a relatively weak correlation between the time constant of $\dot{\mathrm{V}}_{2}$ and that of $\mathrm{HR}(\mathrm{r}=0.50$, Fig 4D). The time constant of HR was significantly shorter than that of $\dot{\mathrm{V}} \mathrm{O}_{2}$ at exercise of low intensity $(\mathrm{p}<0.01)$. The venous lactate concentration $2 \mathrm{~min}$ after exercise was not correlated with the time constant of $\dot{\mathrm{V}} \mathrm{E}(\mathrm{r}=0.18, \mathrm{p}=0.24)$ and showed a weak correlation with the time constants of $\dot{\mathrm{V}} \mathrm{O}_{2}(\mathrm{r}=0.42, \mathrm{p}<0.05)$ and $\dot{\mathrm{V} C O} 2(\mathrm{r}=0.47, \mathrm{p}<0.01)$.

\section{Relationship Between Cardiac Function and Gas Exchange Kinetics}

We divided the 23 subjects into 2 groups, according to their peak $\dot{\mathrm{V}}_{2}$ obtained during preliminary incremental exercise test; patients with peak $\dot{\mathrm{V}} \mathrm{O}_{2} \geq 20 \mathrm{ml} \mathrm{min}^{-1} \mathrm{~kg}^{-1}$ $\left(\mathrm{n}=11,22.6 \pm 3.6 \mathrm{ml} \mathrm{min}^{-1} \mathrm{~kg}^{-1}\right)$ and those with peak $\dot{\mathrm{VO}} 2$ $<20 \mathrm{ml} \mathrm{min}^{-1} \mathrm{~kg}^{-1}\left(\mathrm{n}=12,17.4 \pm 1.4 \mathrm{ml} \mathrm{min}^{-1} \mathrm{~kg}^{-1}\right)$. The time constant of $\dot{\mathrm{V}}_{2}$ during high-intensity exercise was significantly shorter in the patients with higher peak $\dot{\mathrm{V}}_{2}$ as compared with those with a lower peak $\mathrm{VO}_{2}(44.3 \pm 9.7$ vs $56.4 \pm 8.9, \mathrm{p}=0.027)$. The time constants of $\dot{\mathrm{VO}}_{2}, \dot{\mathrm{V}} \mathrm{CO}_{2}$, and 
$\dot{V} E$ during low-intensity exercise also tended to be shorter in the patients with higher peak $\dot{\mathrm{V}}_{2}(0.05<\mathrm{p} \leq 0.1)$. There were no significant differences in the time constants of these variables during recovery after high- or low-intensity exercise between the 2 groups.

\section{Discussion}

The major findings of this study are the following: (1) the time constants of $\dot{\mathrm{V}} \mathrm{O}_{2}, \dot{\mathrm{V}} \mathrm{CO}_{2}$, $\dot{\mathrm{V}} \mathrm{E}$ and HR were significantly prolonged during high-intensity exercise as compared with low-intensity exercise; (2) these kinetics were significantly prolonged during recovery from highintensity exercise as compared with recovery from lowintensity exercise, except for the kinetics of VE; (3) there was no significant difference between the time constant of $\dot{\mathrm{V}} \mathrm{O}_{2}$ during exercise and that of recovery both at exercise of low and high intensity; (4) the time constants of $\dot{\mathrm{V}}_{2}$ and HR were significantly shorter during recovery than during exercise for both the low- and high-intensity exercise; (5) the time constants of $\dot{\mathrm{V}} \mathrm{CO}_{2}$ and $\dot{\mathrm{V}} \mathrm{E}$ were significantly longer than the time constant of $\dot{\mathrm{V}}_{2}$ for both lowand high-intensity exercise and for the recovery of each exercise; (6) there was a significant, but weak correlation between the time constant of $\dot{\mathrm{V}}_{2}$ and $\mathrm{HR}$ during exercise $(\mathrm{r}=0.47)$ and during recovery after exercise $(\mathrm{r}=0.50)$.

\section{Measuring the Time Constants of Gas Exchange Kinetics}

The peak $\dot{\mathrm{V}}_{2}$ at maximal exercise has been considered the gold standard for assessment of exercise capacity in patients with heart failure?3,24 However, the peak $\dot{\mathrm{V}}_{2}$ is not necessarily reproducible? 25 Therefore, interest has focused on the usefulness of the time constants of gas exchange kinetics during submaximal exercise,, $7-13$

If the work rate is mild to moderate, gas exchange kinetics can be characterized by a single exponential equation?,26 During high-intensity exercise, these kinetics become more complex, and a multiexponential fitting equation may be more suitable for analyzing kinetics.26,27 However, we used a single exponential equation to characterize the overall kinetics of $\dot{\mathrm{V}} \mathrm{O}_{2}, \dot{\mathrm{V}} \mathrm{CO}_{2}, \dot{\mathrm{V}}$, and $\mathrm{HR}$ during exercise and recovery for both low- and high-intensity tests in the present study. The methodology involved in the use of a multiexponential equation would be too complex for use in obtaining the clinical parameters of exercise capacity. Recent studies have shown that a single exponential model can be used to characterize these kinetics even during high-intensity exercise, 5

The time constant is equal to the time required to attain approximately $63 \%$ of the asymptotic value? The time constant of $\dot{\mathrm{VO}}$ 2, therefore, represents the rate at which $\dot{\mathrm{V}} \mathrm{O}_{2}$ attains a new steady-state. Because the time constant of $\dot{\mathrm{V}} \mathrm{O}_{2}$ during exercise reflects the kinetics of the increase in cardiac output ${ }^{1-3}$ it has been used to estimate the rapidity of the cardiovascular adaptation to exercise ${ }^{13}$ The usefulness of the kinetics of $\dot{\mathrm{V}} \mathrm{CO}_{2}$ and $\dot{\mathrm{V}} \mathrm{E}$ has also been investigated by several investigators!1,12

\section{Effects of Exercise Intensity on Gas Exchange Kinetics}

In normal subjects, $\dot{\mathrm{V}} \mathrm{O}_{2}$ kinetics are known to become slower with the increasing work rate? In the present study, the intensity of constant work-rate exercise significantly influenced HR and gas exchange kinetics during exercise and recovery in patients with left ventricular dysfunction. The gas exchange kinetics were slower during high-inten- sity exercise, suggesting that cardiovascular and pulmonary responses were delayed during high-intensity exercise in these patients. It might be reasonable that the kinetics of $\dot{\mathrm{V}} \mathrm{CO}_{2}$ and $\dot{\mathrm{V}} \mathrm{E}$ become slower with the increasing intensity, because these kinetics partly depend on the $\dot{\mathrm{V}}_{2}$ kinetics ${ }^{28}$ However, in normal subjects, it has been noted that exercise intensity does not necessarily influence the $\dot{\mathrm{V}} \mathrm{CO}_{2}$ kinetics 6,26 The reason of the discrepancy between these findings in normal subjects and our present findings in cardiac patients is not well understood.

\section{Gas Exchange Kinetics During Exercise and Recovery}

$\dot{\mathrm{V}} \mathrm{O}_{2}$ kinetics during recovery from exercise generally reflected $\dot{\mathrm{V}} \mathrm{O}_{2}$ kinetics during exercise for both low- and high-intensity exercise. However, the kinetics of $\dot{\mathrm{V}} \mathrm{CO}_{2}$ and HR were faster during the recovery from exercise than during the exercise itself. We previously found a sudden increase in stroke volume during early phase of recovery after maximal exercise as compared with that of peak exercise (overshoot phenomenon) in patients with coronary artery disease 29 Thus the faster kinetics of HR during the recovery than during exercise may be attributed to the slowed recovery (overshoot) of stroke volume. Faster kinetics of $\dot{\mathrm{V}} \mathrm{CO}_{2}$ during recovery than during exercise have also been reported in normal subjects, 28,30 although its mechanism has not been clarified.

\section{Correlations Between Heart Rate and Pulmonary Gas Exchange Kinetics}

$\dot{\mathrm{VCO}}_{2}$ is determined mainly by the rate of $\mathrm{O}_{2}$ taken up depending on the muscle substrate for mild to moderate exercise, and additionally by the $\mathrm{CO}_{2}$ derived from the buffering of lactic acid by bicarbonate for high-intensity exercise ${ }^{1}{ }^{1} \dot{\mathrm{V}}_{2}$ and $\dot{\mathrm{V}}_{2}$ kinetics during exercise were correlated in the present study, although $\dot{\mathrm{V}} \mathrm{CO}_{2}$ kinetics were slower than $\mathrm{VO}_{2}$ kinetics. The primary reason for the difference in $\dot{\mathrm{V} C O} 2$ kinetics compared with those of $\dot{\mathrm{V}}_{2}$ is likely due to the alkalizing process associated with the splitting of phosphocreatine 32 in addition to the degree of lactic acidosis and the amount of stored intramuscular $\mathrm{CO}_{2} 33$ The kinetics of $\dot{\mathrm{V}} \mathrm{O}_{2}$ and $\dot{\mathrm{V}} \mathrm{CO}_{2}$ also correlated during recovery.

During moderate exercise, ventilation normally increases at a rate required to remove the additional $\mathrm{CO}_{2}$ generated so that the arterial $\mathrm{PCO}_{2}$ and $\mathrm{pH}$ are usually regulated at close to resting values ${ }^{31}$ Thus, the kinetics of $\dot{\mathrm{VE}}$ and $\dot{\mathrm{V}}_{2} \mathrm{O}_{2}$ were closely related in the present study. The venous lactate concentration $2 \mathrm{~min}$ after exercise, which reflects the development of metabolic acidosis during exercise, showed the strongest correlation with the time constant of VंE during exercise.

Because $\dot{\mathrm{V}}_{2}$ is equal to the cardiac output (stroke volume $\times \mathrm{HR}) \times$ the arterial-mixed venous $\mathrm{O}_{2}$ difference, we expected the kinetics of $\dot{\mathrm{V}}_{2}$ to correlate with the $\mathrm{HR}$ response and hypothesized that the time constant of $\dot{\mathrm{V}}_{2}$ might be estimated from the HR kinetics without the need for measurements of respiratory gases. Thus, we selected only patients with anterior myocardial infarction because both the etiology of cardiac disease and a mixed-site infarction can influence HR kinetics ${ }^{34}$ However, the time constant of $\dot{\mathrm{V}}_{2}$ showed only a weak correlation with the time constant of $\mathrm{HR}$, suggesting that $\mathrm{V}_{2}$ kinetics are also influenced by the rate of change of stroke volume and/or $\mathrm{O}_{2}$ extraction in the exercising muscles in patients with left ventricular dysfunction. 


\section{Clinical Implications for Measuring}

Gas Exchange Kinetics

$\dot{\mathrm{V}} \mathrm{O}_{2}$ kinetics during exercise are thought to be determined by intramuscular events and by the rate of $\mathrm{O}_{2}$ delivery to exercising muscles. A reduction in the blood $\mathrm{O}_{2}$ content $6,5,36$ or the administration of a beta-adrenergicblocking agent ${ }^{15,16}$ is known to slow $\dot{\mathrm{VO}}_{2}$ kinetics. $\mathrm{VO}_{2}$ kinetics were correlated during exercise and recovery in the present study, suggesting that not only the $\mathrm{VO}_{2}$ kinetics during exercise, but also the kinetics during recovery, are useful for estimating the impaired cardiovascular response to exercise and for predicting the degree of improvement as a result of therapy in cardiac patients.

The kinetics of $\dot{\mathrm{V}} \mathrm{CO}_{2}$ during moderate to high-intensity exercise reflect the additional $\mathrm{CO}_{2}$ produced from the buffering of lactic acid by bicarbonate and then excreted from the body by compensatory hyperventilation. Thus, $\dot{\mathrm{V}} \mathrm{CO}_{2}$ kinetics during exercise, in combination with $\dot{\mathrm{V}} \mathrm{O}_{2}$ kinetics, may be useful for estimating the degree of buffering of lactic acid.

Hypoxia speeds and hyperoxia slows $\dot{V}$ E kinetics 37,38 probably via the stimulation or attenuation of the carotid bodies. Although $\dot{\mathrm{V} E}$ was closely related to changes in $\dot{\mathrm{V}} \mathrm{CO}_{2}$ during exercise and recovery, the kinetics of $\dot{\mathrm{V}} \mathrm{E}$ during high-intensity exercise were delayed in the present study. A prolonged time constant of $\dot{V E}$ compared with $\dot{\mathrm{VCO}}_{2}$ suggests transient hypercapnia ${ }^{39}$ and is most likely due to the sensitivity of peripheral chemoreceptors. $\dot{V} E$ kinetics during exercise, as a function of $\dot{\mathrm{V}} \mathrm{O}_{2}$ and $\dot{\mathrm{V}} \mathrm{CO}_{2}$ responses, may therefore be useful for evaluating the sensitivity of chemoreceptors (carotid bodies) and pulmonary function, which limit the rate of the ventilatory response in patients with heart failure 37

The prolongation of $\dot{\mathrm{V} E}$ kinetics during recovery after exercise in cardiac patients ${ }^{11,12}$ indicates a slow recovery of breathing and appears to be related to continuous ventilatory stimuli through the chemoreceptors and other inputs to the respiratory controller. Thus, analysis of $\dot{V} E$ kinetics during recovery may help to clarify the mechanisms of prolonged dyspnea after exercise in patients with heart failure.

\section{Conclusions}

Gas exchange kinetics during exercise and recovery were significantly influenced by the intensity of exercise. The kinetics of gas exchange during the recovery from exercise differed from those obtained during exercise, except for $\dot{\mathrm{V}} \mathrm{O}_{2}$ kinetics. Although future studies are needed to determine how closely the noninvasively determined gas exchange kinetics reflect acid-base regulation and muscle bioenergetics, the present findings provide guidance for the interpretation of gas exchange kinetics in patients with left ventricular dysfunction.

\section{Acknowledgments}

This work was supported in part by a Grant-in-Aid for Scientific Research of the Ministry of Education, Science and Culture of Japan. We appreciate the invaluable assistance of Takasuke Imai, MD, of Tokyo Medical and Dental University.

\section{References}

1. Hughson RL: Exploring cardiorespiratory control mechanisms through gas exchange dynamics. Med Sci Sports Exerc 1990; 22: $72-79$
2. Barstow TJ, Buchthal S, Zanconato S, Cooper DM: Muscle energetics and pulmonary oxygen uptake kinetics during moderate exercise. J Appl Physiol 1994; 77: $1742-1749$

3. Koike A, Hiroe M, Adachi H, Yajima T, Yamauchi Y, Nogami A, et al: Oxygen uptake kinetics are determined by cardiac function at onset of exercise in patients with prior myocardial infarction. Circulation 1994; 90: 2324-2332

4. Linnarsson D: Dynamics of pulmonary gas exchange and heart rate changes at start and end of exercise. Acta Physiol Scand (Suppl) 1974; 415: 1-68

5. Sietsema KE, Daly JA, Wasserman K: Early dynamics of $\mathrm{O}_{2}$ uptake and heart rate as affected by exercise work rate. J Appl Physiol 1989; 67: $2535-2541$

6. Koike A, Wasserman K, McKenzie DK, Zanconato S, Weiler Ravell $\mathrm{D}$ : Evidence that diffusion limitation determines oxygen uptake kinetics during exercise in humans. J Clin Invest 1990; 86: 1698 1706

7. Koike A, Yajima T, Adachi H, Shimizu N, Kano H, Sugimoto K, et al: Evaluation of exercise capacity using submaximal exercise at a constant work rate in patients with cardiovascular disease. Circulation 1995; 91: 1719-1724

8. Sietsema KE, Ben-Dov I, Zhang YY, Sullivan C, Wasserman K: Dynamics of oxygen uptake for submaximal exercise and recovery in patients with chronic heart failure. Chest 1994; 105: 1693-1700

9. Sietsema KE, Cooper DM, Perloff JK, Rosove MH, Child JS, Canobbio MM, et al: Dynamics of oxygen uptake during exercise in adults with cyanotic congenital heart disease. Circulation 1986; 73: $1137-1144$

10. Wasserman K: New concepts in assessing cardiovascular function Circulation 1988; 78: 1060-1071

11. Hayashida W, Kumada T, Kohno F, Noda M, Ishikawa N, Kambayashi M, et al: Post-exercise oxygen uptake kinetics in patients with left ventricular dysfunction. Int J Cardiol 1993; 38: $63-72$

12. Cohen-Solal A, Laperche T, Morvan D, Geneves M, Caviezel B, Gourgon R: Prolonged kinetics of recovery of oxygen consumption after maximal graded exercise in patients with chronic heart failure: analysis with gas exchange measurements and NMR spectroscopy. Circulation 1995; 91: 2924-2932

13. Koike A, Hiroe M, Yajima T, Adachi H, Shimizu N, Kano H, et al: Effects of nicorandil on kinetics of oxygen uptake at the onset of exercise in patients with coronary artery disease. Am J Cardiol 1995; 76: $449-452$

14. Casaburi R, Spitzer S, Haskell R, Wasserman K: Effect of altering heart rate on oxygen uptake at exercise onset. Chest 1989; 95: 6-12

15. Hughson RL, Smyth GA: Slower adaptation of $\mathrm{VO}_{2}$ to steady state of submaximal exercise with beta-blockade. Eur J Appl Physiol 1983; 52: $107-110$

16. Petersen ES, Whipp BJ, Davis JA, Huntsman DJ, Brown HV, Wasserman K: Effects of beta-adrenergic blockade on ventilation and gas exchange during exercise in humans. J Appl Physiol 1983; 54: $1306-1313$

17. Beaver WL, Wasserman K, Whipp BJ: A new method for detecting anaerobic threshold by gas exchange. J Appl Physiol 1986; 60: 2020-2027

18. Sue DY, Wasserman K, Moricca RB, Casaburi R: Metabolic acidosis during exercise in patients with chronic obstructive pulmonary disease. Chest 1988; 94: 931-938

19. Koike A, Weiler-Ravell D, McKenzie DK, Zanconato S, Wasserman $\mathrm{K}$ : Evidence that the metabolic acidosis threshold is the anaerobic threshold. J Appl Physiol 1990; 68: 2521 -2526

20. Lightfoot JT, Tankersley C, Rowe SA, Freed AN, Fortney SM: Automated blood pressure measurements during exercise. Med Sci Sports Exerc 1989; 21: 698-707

21. Hansen JL, Freier EF: Direct assays of lactate, pyruvate, betahydroxybutyrate, and acetoacetate with a centrifugal analyzer. Clin Chem 1978; 24: 475-479

22. Dixon WJ, editor. BMDP statistical software. Berkeley: University of California Press, 1988

23. Franciosa JA, Park M, Levine TB: Lack of correlation between exercise capacity and indexes of resting left ventricular performance in heart failure. Am J Cardiol 1981; 47: 33-39

24. Weber KT, Janicki JS: Cardiopulmonary exercise testing for evaluation of chronic cardiac failure. Am J Cardiol 1985; 55: 22A-31A

25. Janicki JS, Gupta S, Ferris ST, McElroy PA: Long-term reproducibility of respiratory gas exchange measurements during exercise in patients with stable cardiac failure. Chest 1990; 97: 12-17

26. Casaburi R, Barstow TJ, Robinson T, Wasserman K: Influence of work rate on ventilatory and gas exchange kinetics. J Appl Physiol 1989; 67: $547-555$ 
27. Barstow TJ, Mole PA: Linear and nonlinear characteristics of oxygen uptake kinetics during heavy exercise. J Appl Physiol 1991; 71: 2099-2106

28. Grassi B, Marconi C, Meyer M, Rieu M, Cerretelli P: Gas exchange and cardiovascular kinetics with different exercise protocols in heart transplant recipients. J Appl Physiol 1997; 82: 1952-1962

29. Koike A, Itoh H, Doi M, Taniguchi K, Marumo F, Umehara I, et al: Beat-to-beat evaluation of cardiac function during recovery from upright bicycle exercise in patients with coronary artery disease. $A m$ Heart J 1990; 120: 316-323

30. Karlsson H, Lindborg B, Linnarsson D: Time courses of pulmonary gas exchange and heart rate changes in supine exercise. Acta Physiol Scand 1975; 95: 329-340

31. Wasserman K, Hansen JE, Sue DY, Whipp BJ, Casaburi R: Principles of exercise testing and interpretation. Philadelphia: Lea \& Febiger, 1994: 36-42

32. Wasserman K, Stringer WW, Casaburi R, Zhang YY: Mechanism of the exercise hyperkalemia: an alternate hypothesis. J Appl Physiol 1997; 83: 631-643

33. Whipp BJ, Ward SA: Physiological determinants of pulmonary gas exchange kinetics during exercise. Med Sci Sports Exerc 1990; 22:
$62-71$

34. Duilio C, Spinelli L, Liucci GA, Iovino GL, Cuocolo A, Ferro G: Site of myocardial ischemia as a determinant of postexercise blood pressure and heart rate response in coronary artery disease. Am J Cardiol 1993; 72: 1376-1382

35. Springer C, Barstow TJ, Wasserman K, Cooper DM: Oxygen uptake and heart rate responses during hypoxic exercise in children and adults. Med Sci Sports Exerc 1991; 23: 71-79

36. Engelen M, Porszasz J, Riley M, Wasserman K, Maehara K, Barstow TJ: Effects of hypoxic hypoxia on $\mathrm{O}_{2}$ uptake and heart rate kinetics during heavy exercise. J Appl Physiol 1996; 81: 2500-2508

37. Nery LE, Wasserman K, Andrews JD, Huntsman DJ, Hansen JE, Whipp BJ: Ventilatory and gas exchange kinetics during exercise in chronic airways obstruction. J Appl Physiol 1982; 53: 1594-1602

38. Springer C, Barstow TJ, Cooper DM: Effect of hypoxia on ventilatory control during exercise in children and adults. Pediatr Res 1989; 25: $285-290$

39. Whipp BJ, Wasserman K, Casaburi R, Juratsch CE, Weisman ML, Stremel RW: Ventilatory control characteristics of conditions resulting in isocapnic hyperpnea. Adv Exp Med Biol 1978; 99: 355-365 